\title{
La sinovite villonodulare pigmentosa della tibio-tarsica. Storia di un caso clinico
}

A. Guarino ${ }^{1}$, E. Giussani ${ }^{1}$, E. Paresce ${ }^{2}$

${ }^{1}$ III Divisione di Ortopedia e Traumatologia, Istituto Ortopedico G. Pini, Milano; ${ }^{2}$ U. 0 . Reumatologia, Istituto Ortopedico G. Pini, Milano

D01 10.1007/s10261-009-0013-2

ABSTRACT Pigmented villonodular synovitis of the ankle. Case report Pigmented villonodular synovitis is a rarely observed (1.8 cases per million) chronic inflammatory pathology of the sinovia. The aim of this work is to analyse the management of a clinical case with a rare location at the Unit where the Authors work and to study clinical and radiological results one year after treatment with arthroscopic technique.

\section{Introduzione}

La sinovite villonodulare pigmentosa (SVP) è una patologia infiammatoria cronica della membrana sinoviale di raro riscontro ( 1,8 casi per milione), eziopatogenesi tuttora non ben definita e con caratteri anatomo-patologici tipicamente di proliferazione fibroistiocitaria e aggressività locale. La SVP può presentarsi sia in forma localizzata, sia in forma diffusa e può avere sede sia intra- sia extraarticolare [1].

Sede elettiva di localizzazione della forma diffusa, di regola intra- e monoarticolare, è l'articolazione del ginocchio, ma possono essere interessati, con frequenza decrescente, l'anca, la caviglia, il polso e la spalla [2]. La forma extra-articolare di SVP si manifesta generalmente in forma nodulare e viene spesso considerata come un'entità a sé stante con localizzazione tipicamente in corrispondenza delle dita della mano [3]

Scopo del lavoro è analizzare come è stato trattato un caso clinico a localizzazione rara presso l'Unità Operativa dove operano gli Autori.

\section{Materiali e metodi}

Il caso clinico presentato riguarda una paziente di sesso femminile, di 35 anni d'età, sportiva, che da circa sei mesi riferiva artralgie episodiche alla caviglia destra in assenza di eventi traumatici recenti o pregressi. Lieve dolorabilità alle manovre valutative e rigonfiamento articolare sproporzionato rispetto all'entità della sintomatologia cui si associa limitazione della flesso-estensione.

Al fine di ottenere un corretto inquadramento nosologico e una valutazione dell'estensione del quadro patologico abbiamo sottoposto la paziente a risonanza magnetica (RM), esame strumentale che è in grado di dimostrare l'eventuale interessamento di tutte le componenti anatomiche di pertinenza dei tessuti molli articolari (strutture sinoviali, tendinee, legamentose, bursali) e delle strutture ossee.

Alla RM è stato possibile riconoscere un irregolare e diffuso ispessimento della membrana sinoviale dell'articolazione talocrurale, particolarmente spiccato a livello del recesso sinoviale posteriore ove la membrana andava ad assumere aspetto geneamente basso, sia nelle sequenze a corti sia in quelle a lunghi TR (Fig. 1). Si evidenziava inoltre una discreta quantità di versamento che si andava a raccogliere prevalentemente anteriormente. I capi ossei apparivano sostanzialmente regolari per morfologia e struttura con cartilagini di rivestimento sostanzialmente conservate e rapporti corretti. Le altre campo di vista erano regolari analogamente ai legamenti, ai tendini, alle guaine sinoviali di questi ultimi e ai rimanenti rilievi.
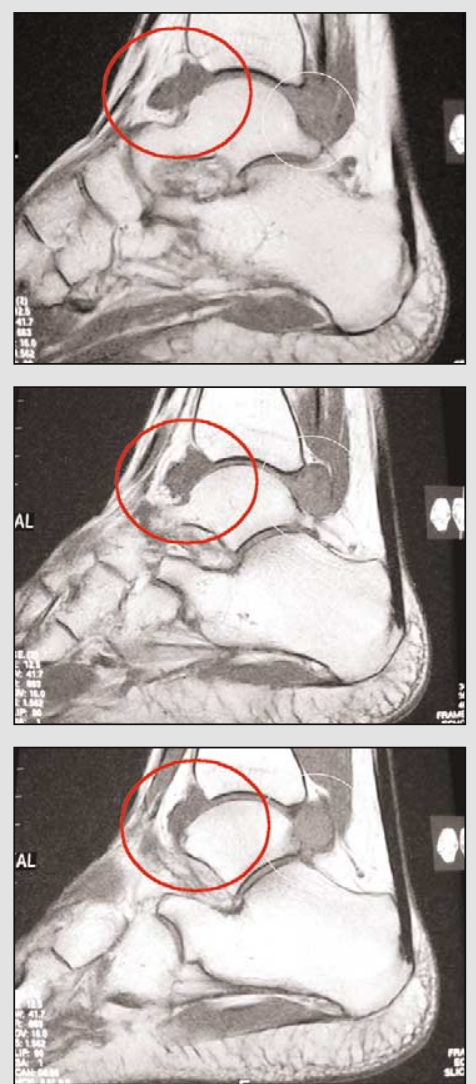

Fig. 1. Quadro RM: è possibile valutare il caratteristico aspetto vegetante della membrana sinoviale che è andata incontro a diffuso ispessimento

In conclusione il quadro RM deponeva per una SVP dell'articolazione talo-crurale.

\section{Tecnica}

A questo punto abbiamo sottoposto la paziente a intervento vegetante, con segnale disomoarticolazioni comprese nel chirurgico in artroscopia per poter osservare direttamente caratteri della lesione, per poter eseguire un prelievo bioptico per il successivo esame istologico e per trattare selettivamente la lesione.

La procedura è stata eseguita attraverso i tradizionali portali artroscopici antero-mediale e antero-laterale, posizionando la paziente in decubito supino in anestesia generale e applicando un laccio pneumostatico a livel lo del terzo inferiore della coscia previo svuotamento del l'arto con fascia di Esmarch All'ispezione del cavo articolare, previa dilatazione con soluzione fisiologica, si è potuto osservare una membrana sinoviale di color ocra prevalentemente nel comparto mediale e nel mortaio tibiale adesa alla cartilagine e tipicamente villiforme (Fig. 2)

Mediante strumento motorizzato e strumento a radiofrequenze abbiamo eseguito una sinovialectomia selettiva con buona rimozione di tessuto patologico, previa asportazione di campioni per l'esame istologico L'intervento è stato concluso con abbondanti lavaggi articolari.

L'esame istologico ha permesso di valutare una sinovite cronica con diffusi accumuli di pigmen to emosiderinico e cellule giganti a tipo corpo estraneo intorno a focolaio di necrosi con cristalli colesterinici. Il reperto era suggestivo di sinovite villonodulare pigmentosa [4]
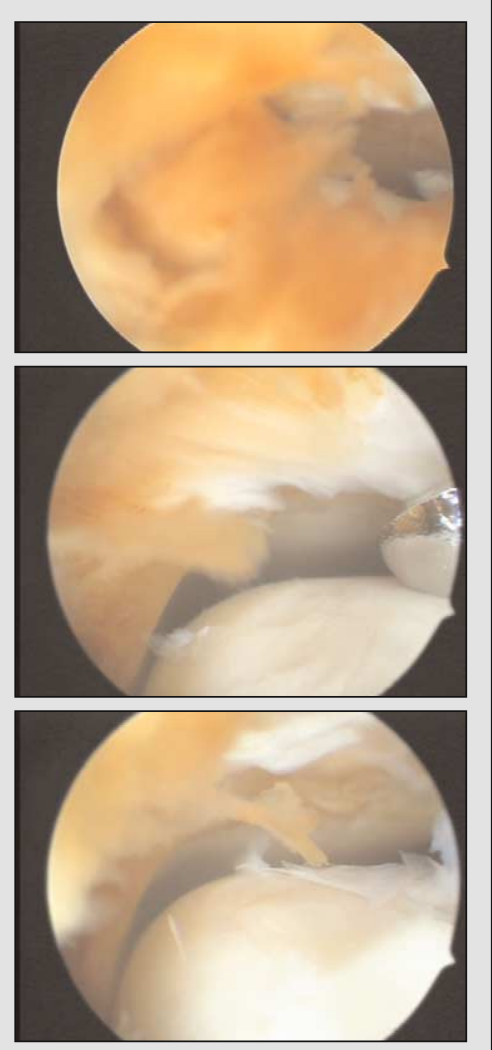

Fig. 2. Reperto intra-operatorio. All'osservazione diretta è possibile rilevare una membrana sinoviale di color ocra prevalentemente nel comparto mediale e nel mortaio tibiale adesa alla cartilagine e tipicamente villiform
Risultati

La paziente ha dimostrato sin dai primi giorni un annullamento della sintomatologia dolorosa a riposo, con decisa riduzione dell'algia al carico anche in ambito dinamico particolarmente espresso.

A distanza di sei mesi dall'intervento la sintomatologia si è completamente risolta, con ripristino della morfologia perilesionale, recupero totale del "range of motion" e ritorno all'attività sportiva amatoriale. A un anno dall'operazione la RMN ha permesso di valutare una stabilizzazione del quadro sinoviale con aree di assoluta normalizzazione specie nella camera anteriore, mentre nel compartimento posteriore persisteva una discreta ipertrofia (Fig. 3)
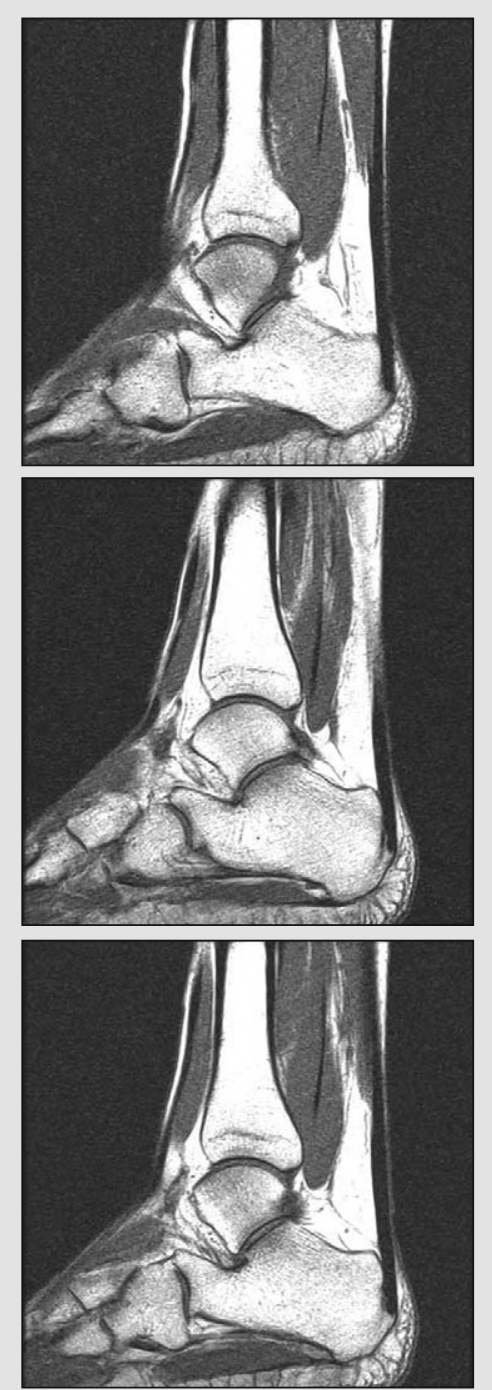

Fig. 3. Controllo RMN a un anno

\section{Discussione}

Restando ferme le considerazioni riguardo la tendenza a recidivare delle sinoviti villonodulari in senso lato, indubbiamente la pratica artroscopica, confortata dall'utilizzo dei nuovi strumenti a radiofrequenza e dalla mininvasività che la caratterizza, appare sicuramente la metodica chirurgica ideale, specie per le piccole articolazioni quali la tibio-tarsica che, se aggredite con tecnica a cielo aperto, sovente vanno incontro a problematiche riabilitative con frequenti rigidità articolari residue. Inoltre proprio il minicoinvolgimento chirurgico rende sicuramente più agevole anche una eventuale ripresa chirurgica.

In particolare l'aggressione per via artroscopica del raro caso di localizzazione tibio-tarsica ha permesso in prima istanza una diagnosi sia morfologica sia istologica precisa e il trattamento immediato e pressoché completo della lesione, favorendo l'asportazione del tessuto patoogico anche nei recessi difficilmente raggiungibili con la pratica chirurgica tradizionale.

Bibliografia

Bouli H, Deppert EJ, Leventhal LJ et al (2004) Pigmented villonodular synovitis: a disease in evolution. J Rheumatol 31:1659-1662

Flandry F, Hughston JC (1987) Pigmented villonodular synovitis. J Bone Joint Surg Am 69:942-949

Granowitz SP, D’Antonio J, Mankin HL (1976) The pathogenesis and long-term end results of pigmented villonodular synovitis. Clin Orthop 114:335-351

4. Darling JM, Glimcher LH, Shortkroff S et al (1994) Expression of metalloproteinases in pigmented villonodular synovitis. Hum Pathol 25:825-830 